\title{
Orbital fluctuations and orbital order below the Jahn-Teller transition in $\mathrm{Sr}_{3} \mathrm{Cr}_{2} \mathrm{O}_{8}$
}

\author{
Zhe Wang, ${ }^{1}$ M. Schmidt, ${ }^{1}$ A. Günther, ${ }^{1}$ S. Schaile, ${ }^{1}$ N. Pascher, ${ }^{1}$ F. Mayr, ${ }^{1}$ Y. Goncharov,,${ }^{1,2}$ D. L. Quintero-Castro, ${ }^{3,4}$ \\ A. T. M. N. Islam, ${ }^{3}$ B. Lake, ${ }^{3,4}$ H.-A. Krug von Nidda, ${ }^{1}$ A. Loidl, ${ }^{1}$ and J. Deisenhofer ${ }^{1}$ \\ ${ }^{1}$ Experimental Physics V, Center for Electronic Correlations and Magnetism, Institute of Physics, \\ University of Augsburg, D-86135 Augsburg, Germany \\ ${ }^{2}$ Institute of General Physics, Russian Academy of Sciences, 119991 Moscow, Russia \\ ${ }^{3}$ Helmholtz-Zentrum Berlin für Materialien und Energie, D-14109 Berlin, Germany \\ ${ }^{4}$ Institut für Festkörperphysik, Technische Universität Berlin, D-10623 Berlin, Germany
}

(Received 28 April 2011; published 23 May 2011)

\begin{abstract}
We report on the magnetic and phononic excitation spectrum of $\mathrm{Sr}_{3} \mathrm{Cr}_{2} \mathrm{O}_{8}$ determined by $\mathrm{THz}$ and infrared (IR) spectroscopy, and electron spin resonance (ESR) measurements across the Jahn-Teller transition, which is detected by specific-heat measurements to occur at $T_{\mathrm{JT}}=285 \mathrm{~K}$. We identify the singlet-triplet excitations in the dimerized ground state and estimate the exchange couplings in the system. ESR absorptions were observed up to $T^{*}=120 \mathrm{~K}$ with a linewidth $\propto \exp \left(-\Delta / k_{B} T\right)$ and $\Delta / k_{B}=388 \mathrm{~K}$, indicating a phonon-mediated spin relaxation via the excited orbital state of the $\mathrm{Cr} e$ doublet in the orbitally ordered state. Upon entering the low-symmetry Jahn-Teller distorted phase below $T_{\mathrm{JT}}$, we find an extended regime $T^{*}<T<T_{\mathrm{JT}}$, where the IR active phonons change only gradually with decreasing temperature. This regime is associated with strong fluctuations in the orbital and lattice degrees of freedom, in agreement with the loss of the ESR signal above $T^{*}$. Using the measured magnetic and phononic excitation spectrum we model the orbital contribution to the specific heat and find the persistence of strong fluctuations far below $T_{\mathrm{JT}}$.
\end{abstract}

DOI: 10.1103/PhysRevB.83.201102

PACS number(s): 71.70.-d, 76.30.-v, 78.30.-j, 78.20.-e

Orbital degrees of freedom (DOF) and their coupling to other DOF play an important role in understanding the physics in many transition-metal compounds, including colossal magnetoresistive manganites, vanadates, and iron-based superconductors. ${ }^{1-3}$ Orbital ordering (OO) mechanisms, ${ }^{4}$ collective orbital excitations, ${ }^{5}$ and frustration effects in the orbital sector have attracted considerable attention and formed the research field of orbital physics. Exotic ground states such as orbital and spin-orbital liquids have been explored both experimentally and theoretically. ${ }^{6}$ Orbital fluctuations play an important role in the formation of these states and may even induce a dimerization of spins via magnetoelastic coupling. ${ }^{7}$

Here we investigate the $\mathrm{OO}$ transition in the spin-gapped dimerized system $\mathrm{Sr}_{3} \mathrm{Cr}_{2} \mathrm{O}_{8}$. This compound has come into focus due to the occurrence of a field-induced magnon condensation. ${ }^{8,9}$ The room-temperature crystal structure of $\mathrm{Sr}_{3} \mathrm{Cr}_{2} \mathrm{O}_{8}$ was determined to be hexagonal with space group $R \overline{3} m .{ }^{10}$ Each $\mathrm{Cr}^{5+}$ ion with spin $S=1 / 2$ is surrounded by an oxygen tetrahedron and couples antiferromagnetically to adjacent $\mathrm{Cr}^{5+}$ ions along the $c$ direction, forming a spinsinglet ground state at low temperatures. The tetrahedral crystal field splits the $3 d$ levels into lower-lying doubly degenerated $e$ and triply degenerated $t$ orbitals. Thus, the $\mathrm{Cr}^{5+}$ ions are Jahn-Teller (JT) active and a JT transition to a monoclinic structure with three twinned domains and space group $C 2 / c$ has been reported by neutron experiments to occur at $275 \mathrm{~K} .^{11,12}$ Primarily associated with the antiferrodistortive displacement of the apical oxygen ions, this transition is suggested to be accompanied by the splitting of the $e$ doublet into a lower-lying $d_{3 z^{2}-r^{2}}$ and excited $d_{x^{2}-y^{2}}$ orbital, and an antiferro-orbital ordering of $d_{3 z^{2}-r^{2}}$ orbitals. In addition, this ordering reportedly changes the magnetic exchange paths and leads to spatially anisotropic exchange couplings. ${ }^{11,12}$ Recent $a b$ initio calculations showed that correlation effects within $\mathrm{Cr}-3 d$ orbitals can account for both the structural phase transition and the singlet ground state. ${ }^{13}$

We present a combined study using $\mathrm{THz}$ and infrared (IR) spectroscopy, electron spin resonance (ESR), and specific heat. We directly observe the singlet-triplet excitations in the ground state and derive the splitting of the $e$ orbitals from the ESR spin relaxation below $120 \mathrm{~K}$. Above $120 \mathrm{~K}$ strong fluctuations are found to influence both the IR active lattice vibrations and the spin relaxation up to the JT transition at $285 \mathrm{~K}$.

Single crystals grown by the floating-zone method ${ }^{12,14}$ were orientated by Laue diffraction and cut along an $a_{h} c_{h}$ plane, where the subscript $h$ stands for hexagonal and a subscript $m$ for monoclinic in the following. Heat capacity was measured in a Quantum Design physical properties measurement system from 1.8 to $300 \mathrm{~K}$. Susceptibility was measured using a superconducting quantum interference device (SQUID) magnetometer (Quantum Design). Polarizationdependent reflectivity was measured from 20 to $300 \mathrm{~K}$ in the far- and mid-IR range using the Bruker Fourier-transform IR spectrometers IFS $113 \mathrm{v}$ and IFS $66 \mathrm{v} / \mathrm{S}$ with a Heflow cryostat (Cryovac). $\mathrm{THz}$ transmission experiments were performed in a Voigt configuration using a Mach-Zehnder-type interferometer with backward-wave oscillators covering the frequency range $115 \mathrm{GHz}-1.4 \mathrm{THz}$ and a magneto-optical cryostat (Oxford/Spectromag) with applied magnetic fields up to $7 \mathrm{~T}$. ESR measurements were performed in a Bruker ELEXSYS E500 cw spectrometer at an $X$-band frequency of $9.48 \mathrm{GHz}$ from 4 to $300 \mathrm{~K}$.

The results of all $\mathrm{THz}$ transmission spectra measured with different frequencies for $\mathbf{H} \| c_{h}$ and $\mathbf{H} \| a_{h}$ are summarized in Fig. 1(d). Absorptions labeled 1, 1', and $3^{\prime}$ in Fig. 1(b) correspond to excitations from the singlet ground state to the excited triplet states that are Zeeman split by the external magnetic field $\mathbf{H}$, while absorption 2 corresponds 

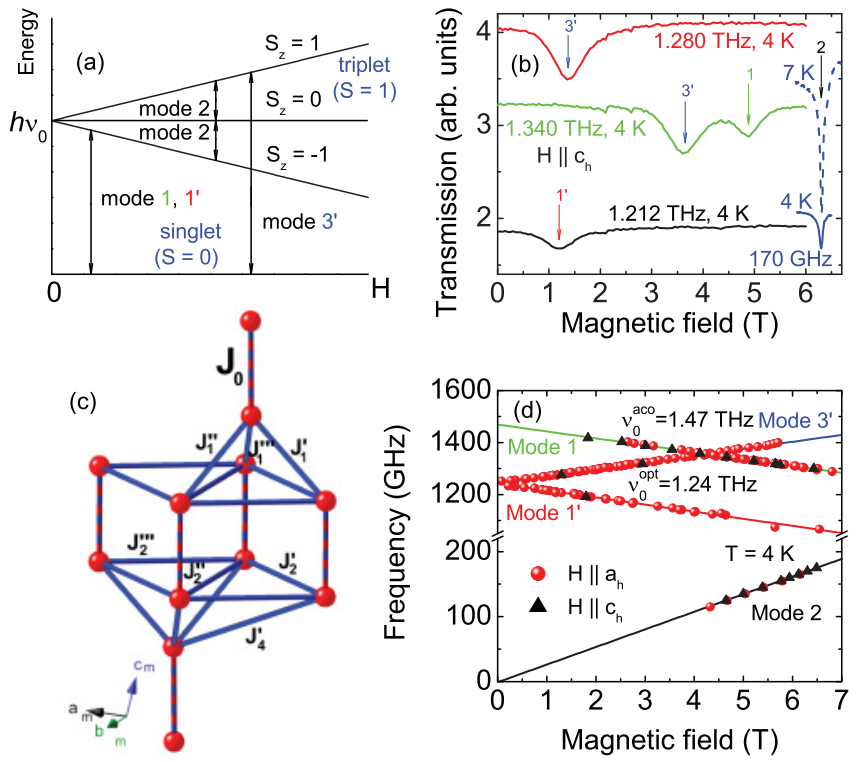

FIG. 1. (Color online) (a) Zeeman splitting of triplet states in a magnetic field. Modes 1, 1', 2, and $3^{\prime}$ are described in the text. (b) Transmission spectra measured at different frequencies at $4 \mathrm{~K}$. The spectrum obtained at $170 \mathrm{GHz}$ and $7 \mathrm{~K}$ (dashed line) is shifted for clarity. (c) Bilayer structure of $\mathrm{Cr}^{5+}$ ions. (d) Magnetic field dependence of the observed absorption frequencies at $4 \mathrm{~K}$ for $\mathbf{H} \| c_{h}$ and $\mathbf{H} \| a_{h}$.

to the intratriplet excitations as illustrated in Fig. 1(a). The excitation spectrum agrees with the one reported for $\mathrm{Ba}_{3} \mathrm{Cr}_{2} \mathrm{O}_{8}$ by Kofu et al., ${ }^{15}$ except for mode $3^{\prime}$ not having been observed for $\mathrm{Ba}_{3} \mathrm{Cr}_{2} \mathrm{O}_{8}$. Modes $3^{\prime}$ and $1^{\prime}$ can be described in terms of a linear Zeeman splitting following $h v=h v_{0} \pm g \mu_{B} H$, with $v_{0}^{\text {opt }}=1.24 \mathrm{THz} \cong 5.13 \mathrm{meV}$ and an effective $g$ factor of $\sim 1.92(3)$, in agreement with the value of 1.94 of mode 2, with ESR data discussed below and reported values for $\mathrm{Ba}_{3} \mathrm{Cr}_{2} \mathrm{O}_{8}$ and $\mathrm{Sr}_{3} \mathrm{Cr}_{2} \mathrm{O}_{8} \cdot{ }^{9}{ }^{15}$ For mode 1 we could observe the lower branch within the available frequency range and found $\nu_{0}^{\mathrm{aco}}=1.47 \mathrm{THz} \cong 6.08 \mathrm{meV}$, which corresponds well to the magnetic excitation energy reported by neutron scattering at the $\Gamma$ point. ${ }^{12}$ Following Ref. 15 , we assign modes 1 and $1^{\prime}$ to the cooperative acoustic $(n=0)$ and optical modes $(n= \pm 1)$ of the coupled dimers of different bilayers with eigenfrequencies $h v_{0} \simeq \sqrt{J_{0}^{2}+J_{0} \gamma}$, where $\gamma=2\left[\left(J_{1}^{\prime}+J_{1}^{\prime \prime}+J_{1}^{\prime \prime \prime}\right) \cos \left(\frac{2}{3} n \pi\right)+\left(J_{2}^{\prime}+J_{2}^{\prime \prime}+J_{2}^{\prime \prime \prime}\right)+\right.$ $\left.\left(J_{4}^{\prime}+J_{4}^{\prime \prime}+J_{4}^{\prime \prime \prime}\right) \cos \left(\frac{2}{3} n \pi\right)\right]$. Here $J_{0}$ denotes the intradimer interaction, $J_{1}, J_{2}$, and $J_{4}$ are the interdimer interactions [see Fig. 1(c)]. Using the values of exchange couplings determined from neutron scattering ${ }^{12}$ we find $h v_{0}^{\text {aco }}=5.92 \mathrm{meV}$ and $h v_{0}^{\mathrm{opt}}=5.14 \mathrm{meV}$, in agreement with our experimental values. The observability of the singlet-triplet transitions 1 , $1^{\prime}$, and $3^{\prime}$ implies an additional anisotropic contribution to the spin Hamiltonian, which mixes singlet and triplet states and relaxes the selection rule $\Delta S .{ }^{16}$ The intensity of mode 2 increases with increasing temperature, in agreement with the thermal population of the excited levels [see absorption lines at 4 and $7 \mathrm{~K}$ in Fig. 1(b)] and corresponds to the expected ESR signal which has been tracked with high sensitivity in a cavity-based setup at $X$-band frequency.

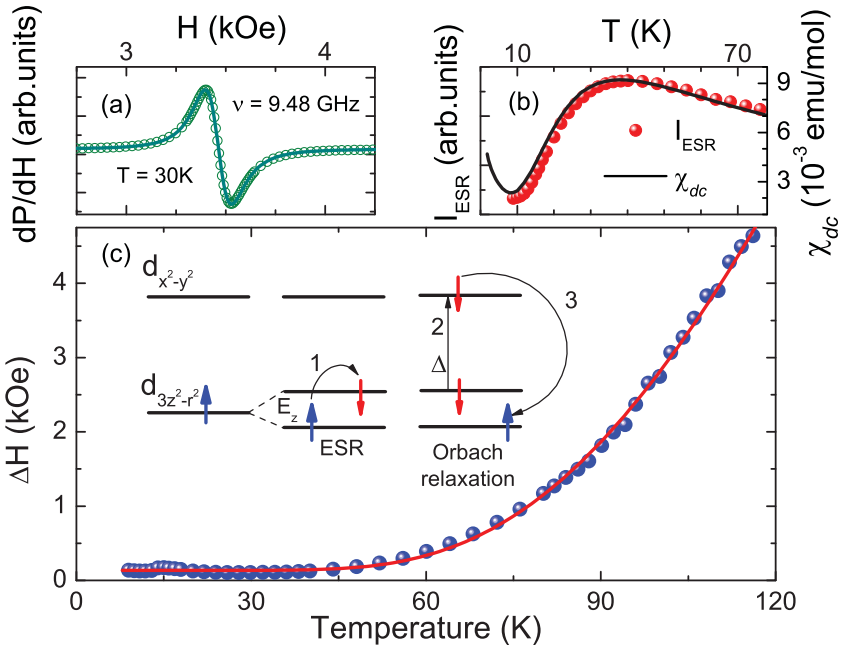

FIG. 2. (Color online) (a) $X$-band ESR spectrum measured at $30 \mathrm{~K}$ for $\mathbf{H} \perp a_{h}$ fitted by a Lorentzian line. Temperature dependence of (b) the ESR intensity $I_{\mathrm{ESR}}$ together with the dc susceptibility $\chi_{\mathrm{dc}}$ measured at $3.4 \mathrm{kOe}$ and (c) of the ESR linewidth. The solid curve is a fit described in the text. Inset: Sketch of the spin relaxation via an Orbach process.

Figure 2(a) shows an ESR derivative absorption spectrum measured at $30 \mathrm{~K}$. The spectrum is well fitted by a derivative Lorentzian line shape characterized by an effective $g$ factor, peak-to-peak linewidth $\Delta H$, and double-integrated intensity $I_{\text {ESR }}$. The latter follows the temperature dependence of the dc susceptibility for $T<T^{*}(=120 \mathrm{~K})$ [see Fig. 2(b)], as expected for an ESR signal originating from the $\mathrm{Cr}^{5+}$ dimers. Above $T^{*}$ the ESR absorption becomes extremely broadened and cannot be tracked any further. In Fig. 2(c) we show the temperature dependence of the linewidth $\Delta H$ with the magnetic field $H \perp a_{h}$. The effective $g$ factor of 1.93(1) is almost constant below $70 \mathrm{~K}$, and is in good agreement with $\mathrm{THz}$ transmission spectra and previous reports. ${ }^{9,15}$ When $\Delta H$ starts to increase strongly and reaches the order of magnitude of the resonance field above $70 \mathrm{~K}$ (see below), the $g$ factor cannot be determined reliably anymore.

The temperature dependence is described using $\Delta H=$ $\Delta H_{0}+A \exp \left(-\frac{\Delta}{k_{B} T}\right)$, with a residual $\Delta H_{0}=135 \mathrm{Oe}, A=$ $130 \mathrm{kOe}$, and a gap $\Delta / k_{B}=388 \mathrm{~K}$. The exponential increase of the ESR linewidth indicates that the relaxation of the excited spins occurs via an Orbach process, ${ }^{17}$ i.e., absorption of a phonon with energy $\Delta$ to an excited orbital state and emission of a phonon with energy $\Delta+E_{Z}$, where $E_{Z}$ is the Zeeman splitting of the lower-lying $e$ orbital, as illustrated in the inset of Fig. 2(c). Thus, the spin dynamics is dominated by spin-lattice relaxation, and we associate the gap with the energy splitting of the $\mathrm{Cr}^{5+} e$ orbitals in the regime $T<T^{*}$, where $\mathrm{OO}$ is complete but the system has not reached its nonmagnetic singlet ground state yet. The splitting $\Delta$ can be associated with the interaction driving $\mathrm{OO}$ and will be important for a theoretical understanding of the energy scales in $\mathrm{Sr}_{3} \mathrm{Cr}_{2} \mathrm{O}_{8} .{ }^{13}$ The fact that ESR spectra cannot be observed above $T^{*}$ is ascribed to a drastically increased spin-lattice relaxation rate for $T>T^{*}$ as a result of strong fluctuations in the orbital and lattice DOF. 


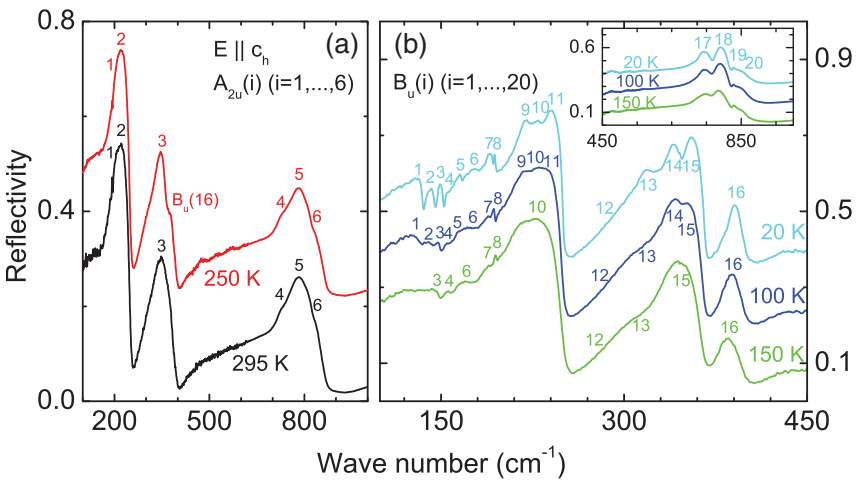

FIG. 3. (Color online) Reflectivity spectra of $\mathrm{Sr}_{3} \mathrm{Cr}_{2} \mathrm{O}_{8}$ with $\mathbf{E} \| c_{h}$ (a) at 295 and $250 \mathrm{~K}$, and (b) at 150, 100, and $20 \mathrm{~K}$. The spectra are shifted with respect to the ones at 150 and $295 \mathrm{~K}$ in order to clearly illustrate the evolution of phonon modes with temperature.

To study the lattice dynamics we performed IR reflectivity measurements with the electric field $\mathbf{E} \| a_{h}$ and $\mathbf{E} \| c_{h}$. Normal mode analysis yields the irreducible representations of IR active modes $6 A_{2 u}(\mathbf{E} \| z)+7 E_{u}[\mathbf{E} \|(x, y)]$ for the hexagonal $R \overline{3} m$ (No. 166) structure. ${ }^{11,18}$ The room-temperature spectra for $\mathbf{E} \| c_{h}$ [shown in Fig. 3(a)] and $\mathbf{E} \| a_{h}$ (not shown) confirm this expectation by exhibiting six modes with eigenfrequencies 192.8, 200.6, 335.6, 734.7, 765.6, and $827.3 \mathrm{~cm}^{-1}$, and seven modes with eigenfrequencies 110.6, 115.6, 183.0, $758.8,805.0,855.1$, and $938.1 \mathrm{~cm}^{-1}$, respectively. For the monoclinic $C 2 / c$ (No. 15b) structure below the JT transition, the number of expected normal modes increases to $19 A_{u}(\mathbf{E} \|$ $y)+20 B_{u}[\mathbf{E} \|(x, z)]$. However, only the selection rule for the $19 A_{u}$ modes is determined by $\mathbf{E} \| b_{m}$, because the unique monoclinic $b_{m}$ axis coincides with a principal axis of the dielectric tensor. The dipole moments of the $20 B_{u}$ modes are confined to the $a_{m} c_{m}$ plane, but their directions do not necessarily coincide with the crystal axes. Using the relations $a_{h}=\frac{1}{2}\left(a_{m}-b_{m}\right), b_{h}=-\frac{1}{2}\left(a_{m}+b_{m}\right)$, and $c_{h}=$ $\frac{3}{2} c_{m}-\frac{1}{2} a_{m},{ }^{11}$ it is clear that upon cooling below $T_{\mathrm{JT}}$ with polarization $\mathbf{E} \| a_{h}$ we may expect to see at least the $19 A_{u}$ modes from the contribution of the monoclinic $b_{m}$ axis plus possible additional $B_{u}$ modes due to the projection of the polarization along $a_{m}$. Similarly, for $\mathbf{E} \| c_{h}$ we can expect to probe the majority of the $20 B_{u}$ modes confined to the $a_{m} c_{m}$ plane. The above transformation corresponds to only one of three reported monoclinic twins ${ }^{12}$ and thus the number of expected normal modes may be even larger. Consequently, one would expect a drastic increase in the number of IR active modes for both measured polarizations when comparing spectra below and above the JT transition, e.g., at 250 and $295 \mathrm{~K}$. However, as shown in Fig. 3(a), the IR spectra do not change dramatically across $T_{\mathrm{JT}}=285 \mathrm{~K}$. Only one additional mode $B_{u}(16)$ is already visible at $250 \mathrm{~K}$, while the expected $20 B_{u}$ modes of the low-temperature structure appear only gradually upon further cooling. This behavior is illustrated in Fig. 3(b), where spectra at 150,100 , and $20 \mathrm{~K}$ are compared. The modes present at $20 \mathrm{~K}$ can be observed at $100 \mathrm{~K}$, while in the phonon spectrum at $150 \mathrm{~K}$ several modes are not resolved anymore and appear to be strongly broadened. A similar behavior has also been observed for the IR spectra measured for $\mathbf{E} \| a_{h}$. Previous structural diffraction studies clearly assign

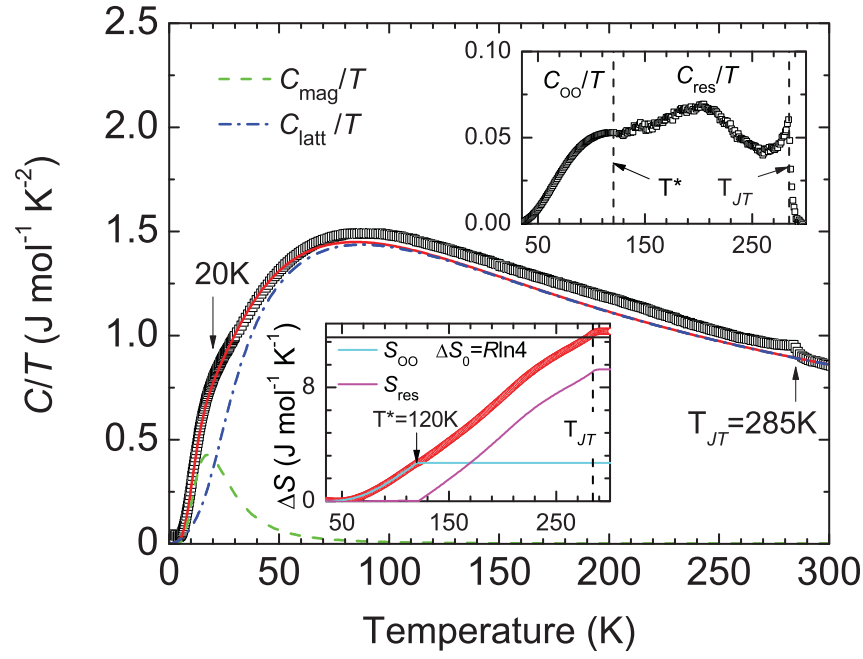

FIG. 4. (Color online) Specific heat divided by temperature $C / T$ vs $T$. The solid line is a superposition of the modeled magnetic (dashed line) and phonon contribution (dashed-dotted line) as described in the text. Upper and lower insets show the temperature dependence of orbital-related specific heat and entropy, respectively.

the monoclinic symmetry to be realized just below $T_{\mathrm{JT}}{ }^{11}$ Consequently, we interpret our results as a signature of strong fluctuations which dominate the lattice dynamics and lead to a strong damping and broadening of phonons in the temperature range $T^{*}<T<T_{\mathrm{JT}}$ with $100 \mathrm{~K}<T^{*}<150 \mathrm{~K}$, in agreement with the ESR results.

Having identified the magnetic and phononic excitations, we turn to the specific heat (see Fig. 4). A peak without any thermal hysteresis, which had not been detected in a previous specific-heat study, ${ }^{19}$ is clearly visible at $T_{\mathrm{JT}}=285 \mathrm{~K}$, indicating an order-disorder phase transition at $285 \mathrm{~K}$. We associate this anomaly with the $\mathrm{OO}$ transition reported to occur at $275 \mathrm{~K}^{11}$ Moreover, an additional broad shoulder is discernible at $\sim 20 \mathrm{~K}$ (see Fig. 4). We assume that the total heat capacity originates from three different parts: a magnetic contribution $C_{\mathrm{mag}}$ corresponding to the thermal population of the excited dimer states, a lattice contribution $C_{\text {latt }}$ due to phonons, and an electronic contribution reflecting the orbital DOF. We approximate the magnetic contribution by $C_{\mathrm{mag}}(T)=N \frac{\partial E}{\partial T}$ using $E=\frac{1}{Z} \sum_{i=0}^{2} g_{i} \epsilon_{i} e^{-\beta \epsilon_{i}}$ with the partition function $Z=\sum_{i=0}^{2} g_{i} e^{-\beta \epsilon_{i}}$, the excitation energies $\epsilon_{0,1,2}=0, h v_{0}^{\text {opt }}, h v_{0}^{\text {aco }}$ as observed in the $\mathrm{THz}$ transmission experiment, degeneracies $g_{0,1,2}=1,2,1$, and $\beta \equiv 1 / k_{B} T$. The resulting magnetic specific heat (dashed line in Fig. 4) accounts well for the shoulder at $20 \mathrm{~K}$. Using the gap $\Delta$ determined by ESR we can fix the orbital contribution $C_{\mathrm{oo}}$ in the completely orbitally ordered phase below $T^{*}$ by a two-level system with splitting $\Delta$, as shown in the upper inset of Fig. 4.

The lattice contribution can be described by a sum of one isotropic Debye $(D)$ and four isotropic Einstein terms $\left(E_{1,2,3,4}\right)$. The ratio between these terms was fixed to $D: E_{1}: E_{2}: E_{3}: E_{4}=1: 3: 4: 3: 2$ to account for the 39 DOF per formula unit. The resulting contribution to the specific heat shown as a dashed-dotted line in Fig. 4 has been obtained with the Debye and Einstein temperatures $\theta_{D}=135.5 \mathrm{~K}, \theta_{E 1}=153.4 \mathrm{~K}, \theta_{E 2}=306.2 \mathrm{~K}, \theta_{E 3}=541.6 \mathrm{~K}$, 
and $\theta_{E 4}=1360 \mathrm{~K}$, consistent with the frequency ranges where IR active phonons of the hexagonal structure occur. Within these constraints the lattice contribution was modeled in such a way that no discontinuity occurs between the Schottky-like term for $T<T^{*}$ and the residual specific heat $C_{\text {res }}=C-C_{\text {mag }}-C_{\text {latt }}-C_{\text {oo }}$ for $T>T^{*}$. In the upper inset of Fig. $4, C_{\text {oo }}$ and $C_{\text {res }}$ are shown together. One can clearly recognize a $\lambda$-shaped anomaly at the JT transition at $285 \mathrm{~K}$ and a broad humplike contribution below the transition followed by the Schottky-like contribution for $T<T^{*}$. The entropy $\Delta S=S_{\mathrm{oo}}+S_{\mathrm{res}}=\int_{0}^{T} d \vartheta\left(C_{\mathrm{oo}}+C_{\mathrm{res}}\right) / \vartheta$ associated with the orbital DOF reaches a value slightly higher than the expected $\Delta S_{0}=R \ln 4$ (the lower inset of Fig. 4). We interpret this observation as due to persistent fluctuations of the orbital and lattice DOF in the temperature range $T^{*}<T<T_{\mathrm{JT}}$, in agreement with the anomalous temperature dependence of the IR phonons and the ESR spectra.
In summary, the Zeeman splitting of singlet-triplet excitations at 5.13 and $6.08 \mathrm{meV}$ was observed in $\mathrm{Sr}_{3} \mathrm{Cr}_{2} \mathrm{O}_{8}$. The spin relaxation is dominated by spin-lattice effects and revealed the splitting $\Delta / k_{B}=388 \mathrm{~K}$ of the low-lying $e$ doublet of the $\mathrm{Cr}$ ions below $120 \mathrm{~K}$. The broadening of spin resonances and polar phonons above $120 \mathrm{~K}$ is ascribed to strong orbital fluctuations. The specific heat clearly marks the JT transition temperature at $285 \mathrm{~K}$ and reveals an extended fluctuation regime below the $\mathrm{JT}$ transition. This indicates the competition of, e.g., spin-orbit coupling and electron-electron interactions with electron-phonon coupling.

We want to thank V. Tsurkan and D. Vieweg for experimental support and P. Lemmens, D. Wulferding, K.-H. Höck, R. M. Eremina, T. P. Gavrilova, and M. V. Eremin for stimulating discussions. We acknowledge partial support by DFG via TRR 80 and FOR 960.
${ }^{1}$ Y. Tokura and N. Nagaosa, Science 288, 462 (2000).

${ }^{2}$ G. Khaliullin, Prog. Theor. Phys. Suppl. 160, 155 (2005).

${ }^{3}$ F. Krüger, S. Kumar, J. Zaanen, and J. van den Brink, Phys. Rev. B 79, 054504 (2009).

${ }^{4}$ K. I. Kugel and D. I. Khomskii, Sov. Phys. Usp. 25, 231 (1982).

${ }^{5}$ E. Saitoh, S. Okamoto, K. T. Takahashi, K. Tobe, K. Yamamoto, T. Kimura, S. Ishihara, S. Maekawa, and Y. Tokura, Nature (London) 410, 180 (2001).

${ }^{6}$ G. Khaliullin and S. Maekawa, Phys. Rev. Lett. 85, 3950 (2000); V. Fritsch, J. Hemberger, N. Büttgen, E.-W. Scheidt, H.-A. Krug von Nidda, A. Loidl, and V. Tsurkan, ibid. 92, 116401 (2004); V. Tsurkan, O. Zaharko, F. Schrettle, Ch. Kant, J. Deisenhofer, H.-A. Krug von Nidda, V. Felea, P. Lemmens, J. R. Groza, D. V. Quach, F. Gozzo, and A. Loidl, Phys. Rev. B 81, 184426 (2010); L. Balents, Nature (London) 464, 199 (2010).

${ }^{7}$ L. F. Feiner, A. M. Oleś, and J. Zaanen, Phys. Rev. Lett. 78, 2799 (1997); G. Jackeli and D. A. Ivanov, Phys. Rev. B 76, 132407 (2007).

${ }^{8}$ T. Nikuni, M. Oshikawa, A. Oosawa, and H. Tanaka, Phys. Rev. Lett. 84, 5868 (2000); C. Rüegg, N. Cavadini, A. Furrer, H.-U. Güdel, K. Krämer, H. Mutka, A. Wildes, K. Habicht, and P. Vorderwisch, Nature (London) 423, 62 (2003); M. Jaime, V. F. Correa, N. Harrison, C. D. Batista, N. Kawashima, Y. Kazuma, G. A. Jorge, R. Stein, I. Heinmaa, S. A. Zvyagin, Y. Sasago, and K. Uchinokura, Phys. Rev. Lett. 93, 087203 (2004); T. Giamarchi, C. Rüegg, and O. Tchernyshyov, Nat. Phys. 4, 198 (2008).
${ }^{9}$ A. A. Aczel, Y. Kohama, C. Marcenat, F. Weickert, M. Jaime, O. E. Ayala-Valenzuela, R. D. McDonald, S. D. Selesnic, H. A. Dabkowska, and G. M. Luke, Phys. Rev. Lett. 103, 207203 (2009).

${ }^{10}$ E. Cuno and H. Müller-Buschbaum, Z. Anorg. Allg. Chem. 572, 95 (1989).

${ }^{11}$ L. C. Chapon, C. Stock, P. G. Radaelli, and C. Martin, e-print arXiv:0807.0877v2.

${ }^{12}$ D. L. Quintero-Castro, B. Lake, E. M. Wheeler, A. T. M. N. Islam, T. Guidi, K. C. Rule, Z. Izaola, M. Russina, K. Kiefer, Y. Skourski, and T. Herrmannsdorfer, Phys. Rev. B 81, 014415 (2010).

${ }^{13}$ G. Radtke, A. Saúl, H. A. Dabkowska, G. M. Luke, and G. A. Botton, Phys. Rev. Lett. 105, 036401 (2010).

${ }^{14}$ A. T. M. Nazmul Islam, D. Quintero-Castro, Bella Lake, K. Siemensmeyer, K. Kiefer, Y. Skourski, and T. Herrmannsdorfer, Cryst. Growth Des. 10, 465 (2010).

${ }^{15}$ M. Kofu, H. Ueda, H. Nojiri, Y. Oshima, T. Zenmoto, K. C. Rule, S. Gerischer, B. Lake, C. D. Batista, Y. Ueda, and S.-H. Lee, Phys. Rev. Lett. 102, 177204 (2009).

${ }^{16}$ V. N. Glazkov, A. I. Smirnov, H. Tanaka, A. Oosawa, Phys. Rev. B 69, 184410 (2004); M. Matsumoto, T. Shoji, and M. Koga, J. Phys. Soc. Jpn. 77, 074712 (2008).

${ }^{17}$ A. Abragam and B. Bleaney, Electron Paramagnetic Resonance of Transition Ions (Clarendon, Oxford, 1970).

${ }^{18}$ E. Kroumova, M. I. Aroyo, J. M. Perez-Mato, A. Kirov, C. Capillas, S. Ivantchev, and H. Wondratschek, Phase Transitions 76, 155 (2003).

${ }^{19}$ Y. Singh and D. C. Johnston, Phys. Rev. B 76, 012407 (2007). 